\title{
Effectiveness of interventions during NICU hospitalization on the neurodevelopment of preterm infants: a systematic review protocol
}

Marilyn Aita ${ }^{1,2^{*}}$, Robyn Stremler ${ }^{3,4}$, Nancy Feeley ${ }^{5,6}$, Andréane Lavallée $^{1}$ and Gwenaëlle De Clifford-Faugère ${ }^{1}$

\begin{abstract}
Background: Previous systematic reviews have examined preterm infants' long-term neurodevelopment after neonatal intensive care unit (NICU) discharge, although none have explored the effectiveness of interventions on preterm infants' neurodevelopment during NICU hospitalization. The aim of this review is to evaluate whether interventions, i.e., sensory stimulation, parental involvement, and control of environment, improve preterm infants' neurodevelopment during their NICU hospitalization.
\end{abstract}

Methods: Experimental studies such as randomized controlled/clinical trials (RCTs) and cluster RCT will be included in this systematic review. Selected studies will be published in English or in French, in the past 15 years from 2002 to 2017. The following electronic databases will be searched to locate relevant studies: CINAHL, MEDLINE, PubMed, EMBASE (OVID), Cochrane Database of Systematic Reviews, Cochrane Central Register of Controlled Trials (CENTRAL), and Web of Science. For all steps in selecting studies, agreement will be reached between two experts in neonatology. Data extraction will be performed independently by the two same experts and will then be compared. The Cochrane assessment tool will be used to screen the studies for risk of bias. A meta-analysis will be performed if the included studies are sufficiently homogeneous. Results will be analyzed using a standardized mean difference (with a 95\% confidence interval). Statistical heterogeneity will be evaluated using the $x^{2}$ test at the significance level of 0.1 and the $P^{2}$ with the classification suggested by PRISMA-P. If possible, subgroup analyses will be carried out considering preterm infants' gestational age, length of NICU hospitalization, and the characteristics of the intervention such as who delivered it, the type, the dose, the frequency, and the duration. Data synthesis will be performed using the RevMan 5.1 software. Publication bias and selection of variables in publication will be examined using the graphical method of funnel plot and with the statistical test of Egger. Quality of the evidence of all outcomes will be assessed using the Grades of Recommendations Assessment, Development and Evaluation (GRADE) Working Group.

Discussion: The results of this systematic review will highlight which interventions are effective for promoting preterm infants' neurodevelopment during NICU hospitalization and will contribute to the body of knowledge in neonatal care by providing guidance for NICU clinical practice and research.

Systematic review registration: PROSPERO CRD42017047072

Keywords: Preterm infants, Neurodevelopment, NICU, Interventions, Hospitalization

\footnotetext{
* Correspondence: marilyn.aita@umontreal.ca

${ }^{1}$ Faculty of Nursing, Université de Montréal, Montréal, Canada

${ }^{2}$ Research Center of the CHU Sainte-Justine, Montréal, Canada

Full list of author information is available at the end of the article
}

(c) The Author(s). 2017 Open Access This article is distributed under the terms of the Creative Commons Attribution 4.0 International License (http://creativecommons.org/licenses/by/4.0/), which permits unrestricted use, distribution, and reproduction in any medium, provided you give appropriate credit to the original author(s) and the source, provide a link to the Creative Commons license, and indicate if changes were made. The Creative Commons Public Domain Dedication waiver (http://creativecommons.org/publicdomain/zero/1.0/) applies to the data made available in this article, unless otherwise stated. 


\section{Background}

Because of an interruption of intrauterine growth and a neonatal intensive care unit (NICU) hospitalization, preterm infants' are more susceptible to encounter significant short- and long-term neurodevelopmental impairments. In fact, a recent epidemiological study reveals that over $25 \%$ of infants born between 28 and 32 weeks of gestation have neurodevelopmental disabilities such as cognitive, motor, visual, or hearing deficits, at the age of 2 years, and this proportion increases by $15 \%$ at age ten to reach around $40 \%$ [1].

The preterm infants' neurodevelopment is influenced during the NICU hospitalization where the experiences they encounter can have significant consequences for the development and functioning of their brain [2, 3, 4]. While hospitalized in the NICU, the infant's brain goes through a period of critical growth between 24 and 40 weeks of gestation $[4,5]$. A series of complex neurological events occur, such as the establishment of synaptic and neuronal connections, as well as the proliferation of significant structures like the thalamus, the cortex, and the cerebellum, all vulnerable to exogenous and endogenous experiences [4]. There is consensus that NICU-related factors which influence the preterm infants' neurodevelopment during hospitalization are, among others, environmental stimulation [6-11], interactions between parents and infants [10-12], and the caregiving experience [5]. Hence, interventions aimed at sustaining or modifying these NICU factors during hospitalization should promote preterm infants' optimal neurodevelopment.

The effectiveness of interventions initiated during the NICU hospitalization has been evaluated in previous systematic reviews on preterm infants' health outcomes. Those interventions include developmental care [13], NICU noise reduction [14], skin-to-skin contact [15], and early interventions involving parents [16]. Although these reviews have all included preterm infants' long-term neurodevelopment after discharge (i.e., from 12 to 24 months of age) as an outcome, none specifically examined the effectiveness of such interventions on the preterm infants' neurodevelopment during their NICU hospitalization. Identifying effective interventions promoting preterm infants' neurodevelopment as soon as they are hospitalized in the NICU will guide clinical neonatal clinical care and may foster preterm infants' long-term development. Moreover, the results of this systematic review will provide a better understanding of the effectiveness of early interventions on the underlying processes of preterm infants' neurodevelopment during hospitalization in addition to guiding neonatal research.

\section{Objective}

The objective of this systematic review is to evaluate whether interventions implemented in the NICU improve preterm infants' neurodevelopment during their hospitalization. This systematic review is guided by the following question: During NICU hospitalization, what is the effectiveness of interventions on the neurodevelopment of preterm infants receiving these interventions compared to those who did not receive these interventions?

\section{Methods}

The protocol for this systematic review was developed according to the Preferred Reporting Items of Systematic Reviews and Meta-Analysis for Protocols (PRISMA-P) 2015 [17]. The PRISMA checklist is provided as an additional file to the protocol (see Additional file 1). The systematic review protocol has been registered in the International Prospective Register of Systematic Reviews (PROSPERO): CRD42017047072.

\section{Eligibility criteria of the selected studies}

The studies selected in this systematic review will meet the following eligibility criteria which are described according to the study design (including publication, language, and year) participants, interventions, comparators, and outcomes.

\section{Study design}

Experimental studies such as randomized controlled/clinical trials (RCTs) and cluster RCTS will be included. We will exclude studies where the randomization process was not random, i.e., assigning alternatively participants, first half of participants to one group or first NICU receiving the intervention compared to a second one not receiving the intervention or assignment of participants by characteristic such as date of birth. We will also exclude quasiexperimental studies (no randomization or no comparative group), as well as all descriptive and case-control studies. Only including RCTs in this systematic review is justified by the fact that this research design provides the highest level of evidence. Accordingly, strongest inferences can be concluded from systematic reviews which include RCTs with reliable findings [18]. Selected studies will be written in English or in French and will have been published in the past 15 years, from 2002 to 2017.

\section{Participants}

Studies including preterm infants, born between 24 to 36 6/7 weeks of gestation will be considered. Infants with major brain abnormalities or any other health situation influencing neurodevelopment, such as intraventricular hemorrhage greater than grade II or brain malformation, will be excluded. 


\section{Interventions}

All interventions, aimed either at preterm infants or parents, instigated during NICU hospitalization will be considered. These interventions can be delivered either by healthcare professionals or parents or by both. For example, those may include either sensory stimulation, parental involvement, control of environment. Multifaceted interventions will be excluded from this review as it will be difficult to estimate which component was more efficacious in promoting infants' neurodevelopment and will preclude meta-analysis if applicable. If indicated, we will contact authors to obtain details about the interventions.

\section{Comparator}

All types of comparator groups, such as non-exposed control group or a group exposed to different intervention, will be included in this systematic review.

\section{Outcome}

Studies reporting preterm infants' neurodevelopment as an outcome evaluated with a standardized instrument, scale, or test during the NICU hospitalization will be included in this systematic review. Examples of standardized instruments, scales, and tests measuring neurodevelopment are the Assessment of the Preterm Infants Behaviors (APIB) [19], NICU Network Neurobehavioral Scale [20], General Assessment Movement (GMA) [21], and electroencephalography (EEG).

\section{Search strategy}

An expert librarian will be consulted to create a list of keywords to be used individually or in combination to conduct the search. The following electronic databases will be searched with $\mathrm{MeSH} / \mathrm{Thesaurus}$ terms to screen for relevant studies for this systematic review: CINAHL, MEDLINE, PubMed, EMBASE (OVID), Cochrane Database of Systematic Reviews, Cochrane Central Register of Controlled Trials (CENTRAL), and Web of Science (see Additional file 2 for an example of a search strategy in MEDLINE). The Scopus database will also be reviewed to search for trials in conference proceedings or in Proquest for thesis and dissertations. We will also look at the clinicaltrials.gov website to identify trials which are presently underway. The reference list of studies identified for this review will be screened to identify other potential studies.

\section{Screening of studies}

All references of studies selected for this review will be managed in the EndNote $\odot$. All duplicates of studies will be identified by two separate reviewers who are experts in neonatology and then compared to ensure appropriate deletion of studies. These two reviewers will then screen the remaining studies for eligibility in two steps. The first step will consist of reviewing all studies' titles and abstracts to identify which studies meet the eligibility criteria. The second step will consist of reviewing the studies' full-text reports to evaluate their appropriateness to be included in the systematic review. For all steps, i.e., deleting duplicates and reviewing titles/abstracts and then full-text papers, an agreement will be reached between the two reviewers. In case of disagreements at any of these steps, a third expert will be consulted. Numbers of studies reviewed, included, and excluded and reasons for exclusion will be noted in a report form.

\section{Data extraction}

Data extraction will be performed independently by two experts and will then be compared. A pilot of data extraction will be done with ten studies. The extracted information will be recorded including the study title, the trial number, the authors and publication year, the country of publication, the research design, the sample size, the description of the intervention (name, who delivered it, comparator, dose, frequency, timing, duration, followup), sample (gestational age, age at assessment, weight at birth, gender, severity of illness score), timing of assessment, and outcome, including the definition of neurodevelopment as well as the instruments, scales, or tests used to measure this outcome. Multiples reports of the same study will only be included if data reported are different in each report. All extracted data will be recorded in RevMan 5.1 .

\section{Assessment of risk of bias}

The Cochrane's risks of bias [22] assessment tool will be used to screen the studies' risk of bias which will be classified as low, high, or unclear risk of bias. The tool screens for sequence generation, allocation concealment, blinding, incomplete outcome data, and selective outcome reporting. Risk of bias will be assessed independently by two reviewers. A third expert will be involved if a disagreement cannot be solved among the two other experts.

\section{Data synthesis}

A meta-analysis will be performed if the included studies are sufficiently homogeneous.

\section{Measuring treatment effect}

The results will be analyzed using a standardized mean difference (with a 95\% confidence interval) since different tools measuring preterm infants' neurodevelopment will be considered in this review. 


\section{Missing data}

Authors of studies will be contacted to obtain missing data; if data cannot be recovered, then a method of permutation will be used.

\section{Assessment of heterogeneity}

The heterogeneity of the studies will be evaluated using the $X^{2}$ test at the significance level of 0.1 and the $I^{2}$ with the classification suggested by the PRISMA-P: 0 to $40 \%$ not important heterogeneity, 30 to $60 \%$ moderate heterogeneity, 50 to $90 \%$ considerable heterogeneity, and $75 \%$ $100 \%$ significant heterogeneity [17]. Considerable heterogeneity $>50 \%$ will be explored using subgroup analysis.

\section{Subgroup analysis}

If possible, subgroup analysis will be carried out considering the preterm infants' gestational age (GA) at birth and at assessment, the length of hospitalization, the characteristics of the intervention such as who delivered it, the type, dose, frequency, and duration.

\section{Data synthesis}

Data synthesis will be performed using the RevMan 5.1 software. If the heterogeneity of the studies is not significant, a fixed effect with the Mantel-Haenszel method [23] will be chosen for data synthesis; if the studies' heterogeneity is considerable (i.e., $X^{2}, p>0.1$ and $I^{2} \geq 50 \%$ [17]), the data synthesis will only be narrative.

\section{Meta-analysis of bias}

Publication bias and selection of variables in publication will be examined using the graphical method of funnel plot if $>10$ studies are included in this review and with the statistical test of Egger [24]. In the presence of bias, even small, a random estimation will be necessarily selected for data synthesis.

\section{Quality of evidence}

The quality of the evidence for all outcomes will be assessed using the Grades of Recommendations Assessment, Development and Evaluation (GRADE) Working Group, which is considered a valid approach [25].

\section{Discussion}

The results of this systematic review will highlight which interventions are effective for promoting preterm infants' neurodevelopment during NICU hospitalization and will guide neonatal clinical care accordingly. Implementing effective interventions as soon as preterm infants are in the NICU will promptly optimize their neurodevelopment and may prevent further long-term neurological impairments in this vulnerable population. Findings of this systematic review will also highlight the state of knowledge on interventions which may only be effective during NICU hospitalization and will guide neonatal research with recommendations. Findings of this review will be published in a scientific journal and will contribute to the body of knowledge in neonatal care by providing guidance for the NICU clinical practice and neonatal research.

\section{Additional files}

Additional file 1: PRISMA-P 2015 Checklist. (PDF 249 kb)

Additional file 2: MEDLINE search strategy. (PDF 181 kb)

Abbreviations

GA: Gestational age; NICU: Neonatal intensive care unit

\section{Acknowledgements}

The authors would like to thank Chantal Roy from the Applied Clinical Research Unit (URCA) of the CHU Sainte-Justine for information guiding the writing of this protocol and Assia Mourid, a librarian at the Faculty of Nursing, Université de Montréal, and Josianne Dupuis, a librarian for supervising the search strategy for this review. Nancy Feeley is supported by a Senior Research Scholar Career Award from the le Fonds de recherche du Québec - Santé (FRQS).

\section{Funding}

This systematic review is supported by the Quebec Network on Nursing Intervention Research (RRISIQ).

Availability of data and materials

Not applicable.

\section{Authors' contributions}

MA wrote the initial protocol. RS, NF, AL, and GDC all contributed to the revision of the protocol to be submitted for publication. All authors have read and approved the final format of this protocol.

Ethics approval and consent to participate

Not applicable.

Consent for publication

Not applicable.

Competing interests

The authors declare that they have no competing interests.

\section{Publisher's Note}

Springer Nature remains neutral with regard to jurisdictional claims in published maps and institutional affiliations.

\section{Author details}

${ }^{1}$ Faculty of Nursing, Université de Montréal, Montréal, Canada. ${ }^{2}$ Research Center of the CHU Sainte-Justine, Montréal, Canada. ${ }^{3}$ Lawrence S. Bloomberg Faculty of Nursing, University of Toronto, Toronto, Canada. ${ }^{4}$ Hospital for Sick Children (SickKids), Toronto, Canada. ${ }^{5}$ Ingram School of Nursing, McGill University, Montréal, Canada. ${ }^{6}$ Centre for Nursing Research and Lady Davis, Jewish General Hospital, Montréal, Canada.

Received: 7 February 2017 Accepted: 19 October 2017

Published online: 03 November 2017

References

1. Johnston KM, Gooch K, Korol E, Vo P, Eyawo O, Bradt P, Levy A. The economic burden of prematurity in Canada. BMC Pediatr. 2014;14:1-10.

2. Altimier L, Kenner C, Damus K. The Wee Care Neuroprotective NICU Program (Wee Care): the effect of a comprehensive developmental care training program on seven neuroprotective core measures for familycentered developmental care of premature neonates. Newborn Infant Nurs Rev. 2015;1:6-16. 
3. Head LM. The effect of kangaroo care on neurodevelopmental outcomes in preterm infants. J Perinat Neonatal Nurs. 2014;28:290-9.

4 Volpe JJ. Brain injury in premature infants: a complex amalgam of destructive and developmental disturbances. Lancet Neurol. 2009:8:110-24.

5 Pickler RH, McGrath J, Reyna BA, McCain N, Lewis M, Cone S, Wetzel P, Best A. A model of neurodevelopmental risk and protection for preterm infants. J Perinat Neonatal Nurs. 2010;24:356-65.

6 Aita M, Snider LM. The art of providing developmental care in the NICU: a concept analysis. J Adv Nurs. 2003;41:223-32.

7 Lui WF, Laudert S, Perkins B, Macmillan-York E, Martin S, Graven S, NIC/Q 2005 Physical Environment Exploratory Group. The development of potentially better practices to support the neurodevelopment of infants in NICU. J Perinatol. 2007;27:S48-74.

8 Chaudari S. Neonatal intensive care practices harmful to the developing brain. Indian Pediatr. 2011;48:437-40.

9 Ramachandran S, Dutta S. Early developmental care interventions of very low birth weight infants. Indian Pediatr. 2013;50:765-70.

10 Santos J, Pearce SE, Stroustrup A. Impact of hospital-based environmental exposures on neurodevelopmental outcomes of preterm infants. Curr Opin Pediatr. 2015;27:254-60.

11 Xiong T, Gonzalez F, Mu D-H. An overview of risk factors for poor neurodevelopmental outcomes associated with prematurity. World J Pediatr. 2012;8:293-300.

12 Milgrom J, Newnham C, Anderson PJ, Doyle LW, Gemmill AW, Lee K, Hunt RW, Bear M, Inder T. Early sensitivity training for parents of preterm infants: impact on the developing brain. Pediatr Resh. 2010;67:330-5.

13 Symington A, Pinelli J. Developmental care for promoting development and preventing morbidity in preterm infants. Cochrane Database of Syst Rev. 2009;19:CD001814.

14 Almadhoob A, Ohlsson A. Sound reduction management in the neonatal intensive care unit for preterm or very low birthweight infants. Cochrane Database of Syst Rev. 2015;1:CD010333.

15 Conde-Agudelo A, Diaz-Rossello J. Kangaroo mother care to reduce mortality and morbidity in low birthweight infants. Cochrane Database of Syst Rev. 2016;23:CD 002771.

16 Vanderveen JA, Bassler D, Robertson CM, Kirpalani H. Early interventions involving parents to improve neurodevelopmental outcomes of premature infants: a meta-analysis. J Perinatol. 2009;29:343-51.

17 Shamseer L, Moher D, Clarke M, Ghersi D, Liberati A, Petticrew M, Shekelle P, Stewart LA, the PRISMA-P Group. Preferred Reporting Items for Systematic Review and Meta-Analysis Protocols (PRISMA-P) 2015: elaboration and explanation. BMJ. 2015;349:1-25.

18 Charrois TL. Systematic reviews: what do you need to know to get started? Can J Hosp Pharm. 2015;68(2):144-8.

19 Als H, Butler S, Kosta S, McAnulty G. The Assessment of Preterm Infants' Behavior (APIB): furthering the understanding and measurement of neurodevelopmental competence in preterm and full-term infants. Ment Retard Dev Disabil Res Rev. 2005;11(1):94-102.

20 Lester B, Tronick EZ. History and description of the neonatal intensive care unit network neurobehavioral scale. Pediatrics. 2004;113(3):634-40.

21 Einspieler C, Prechtl HF. Prechtl's assessment of general movements: a diagnostic tool for the functional assessment of the young nervous system. Ment Retard Dev Disabil Res Rev. 2005;11(1):61-7. https://doi.org/10.1002/ mrdd.20051.

22 Higgins JPT, Green S (editors). Cochrane Handbook for Systematic Reviews of Interventions Version 5.1.0 [updated March 2011]. The Cochrane Collaboration, 2011. Available from http://handbook.cochrane.org.

23 Mantel N, Haenszel MW. Statistical aspects of thee analysis of data from retrospective studies of disease. J Nat Cancer Inst. 1959;22:719-48.

24 Egger M, Davey Smith G, Schneider M, Minder C. Bias in meta-analysis detected by a simple, graphical test. BMJ. 1997:315:629-34.

25 GRADE Working Group. Grading quality of evidence and strength of recommendations. BMJ. 2004:328:1-8. 